\title{
Serpentine Robot Model and Gait Design Using Autodesk Inventor and Simulink SimMechanics
}

\author{
Daniel $^{1}$, Mohammad Iman Alamsyah ${ }^{1}$, Erwin $^{1}$, and Sofyan Tan ${ }^{1}$ \\ ${ }^{1}$ Universitas Bina Nusantara, Computer Engineering Department, Jakarta, Indonesia
}

\begin{abstract}
The authors introduce gaits of a serpentine robot with linear expansion mechanism where the robot varies its length using joints with three degrees of freedom. The 3D model of the serpentine robot is drawed in Autocad Inventor ${ }^{\circledR}$ and exported to SimMechanics ${ }^{\circledR}$ for straighforward modeling of the kinematics. The gaits are important for robots designed to explore ruins of disasters where the working spaces are very tight. For maximum flexibility of the serpentine robot, we adopted a joint design with three parallel actuators, where the joint is capable of linear movement in the forward axis, and rotational movements around two other axes. The designed linear expansion gaits is calculated for forward movement when the robot is posing straight or turning laterally.
\end{abstract}

\section{Introduction}

Natural disasters such as earthquake, landslide, and human-caused disasters such as fire, explosion, collapsing building, etc. in urban places often cause human victims to be trapped under ruins. When they occur the urban search and rescue (USAR) teams are always confronted with a very sort window of time to search and rescue survivors. Searching for survivors most of the time are also hampered by the difficulty to enter or remove the vast amount rubbles. In a worst case scenario such as in the rubble of the Word Trade Center on September $11^{\text {th }} 2011$ most of the voids were less than $1 \mathrm{~m}$ diameter and more than $2 \mathrm{~m}$ deep into it, which are impossible for the USAR teams to enter [1]. Even if the size of the voids are possible for USAR teams to enter, they still have to worry about the stability of the rubbles on top the voids.

In such situations the deployment of mobile robots can be very helpful in the search of victims. However various variables of the mobile robots should be considered, such as the size, the shape, the moving mechanism, the communication, the sensor, and the control of the robot [1]. The basic consideration is the size, shape, and moving mechanism of the mobile robot. The size of the robot should be small enough to be transported and inserted into the voids easily. The shape and moving mechanism of the mobile robot should be effective againts very irregular surfaces in the rubles and should be able to right itself when flipped.

Snake-shaped mobile robot suit the irregular shape of void in a rubble. Its slender and flexible body allows it to adapt to various shapes of voids. Its long shaped body allow it to climb much higher than its height and to move over crevices. The large number of segments of the a robot also makes it more reliable in dealing with failing segments [2].

The moving mechanisms using wheels or legs are prone to jamming when the rubbles contain debris small enough to slip into the rotors or joints [3]. However slithering movement of a serpentive robot uses no wheel or leg which increase its reliability for deployment in ruins of urban disasters.

Between various locomotion strategies of snakes, the rectilinear progression is the most space conserving gait where it does not need to bend its body laterally to move forward. The rectilinear progression makes use of the scales on belly of the snake to pull its body forward [2]. This gait only need the snake to lift its body slightly in order to place its belly forward, hence its best for movement in space-confined voids.

We proposed a snake-shaped (serpentine) robot having the ability to expand its lengh between segments that allow it to do rectilinear progression for forward movement gait. This moving mechanism allows the robot to have the whole body covered with skin to protect is against water, small particles, or even heat, which is not possible with robot with wheel or tread such as in [4].

\section{Model of the serpentine robot}

The serpentine robot is a 3-DOF hyper-redundant robot adopted from [5]. The shape of the serpentine robot is modelled in Autocad Inventor ${ }^{\circledR}$ software. The 3D model 
of the robot is then exported to Simulink ${ }^{\circledR}$ (MATLAB $^{\circledR}$ ) in order to model the kinematic of the robot using SimMechanics ${ }^{\circledR}$ add-on. The kinematic model in Simulink ${ }^{\circledR}$ can be driven to evaluate the gait of serpentine robot.

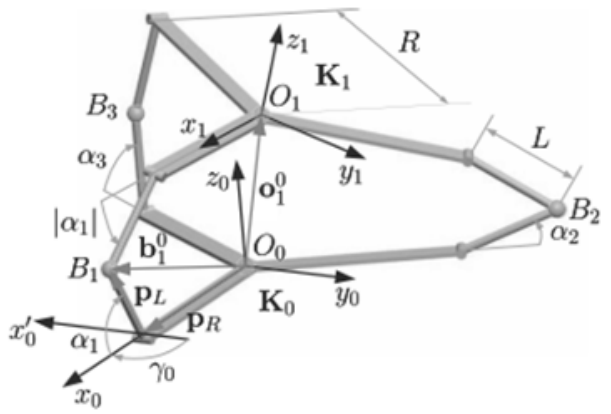

Fig. 1. A segment design of the serpentine robot (adopted from $[5])$.

The design of each segment of the serpentine robot, adopted from [5], is shown in Fig 1. The labels are described below:

- $K_{0}$ : lower plate frame

- $K_{1}$ : upper plate frame

- $R$ : radius of each plate

- $L$ : length of linkage between plates

- $B_{\mathrm{i}}:$ spherical joint $i$ at folding linkage $i$

- $O_{0}$ : origin of lower plate frame

- $O_{1}$ : origin of upper plate frame

- $\alpha_{\mathrm{i}}$ : angle between linkage $i$ and plate

The serpentine robot has 4 segments, each formed by a circular upper plate and a circular lower plate. The two plates are supported by three folding linkages distributed evenly at 120 degree from each others, each shown in Fig 2. The choice of 120 degree is trivial to simplify the mathematical model and the construction.

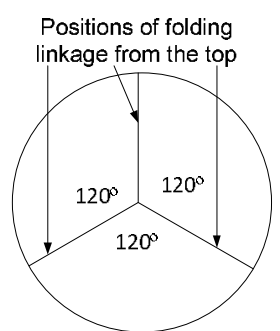

Fig. 2. Each segment looking through the upper plate, showing the folding linkage (adopted from [5]).

Each folding linkage that connect the upper and lower plate is consisted of the upper and lower linkages. The upper and lower linkage is connected by a spherical joint. Each linkage is connected to the plate by a revolute joint.

3D model of a segment in Autodesk Inventor is shown in Fig 3, while 3D model of a 4-segments serpentine robot is shown in Fig 4. The 3D model of the serpentine robot is exported to SimMechanics ${ }^{\circledR}$ using the SMLink add-on in the Autodesk Inventor.

Portion of the resulting model in SimMechanics ${ }^{\circledR}$ is shown in Fig 5, where plate 2 is connected to plate 1 through three linkages (Link 1A, 1B, and 1C), and plate 3 is connected to plate 2 also through three folding linkages (Link 2A, 2B, and 2C).

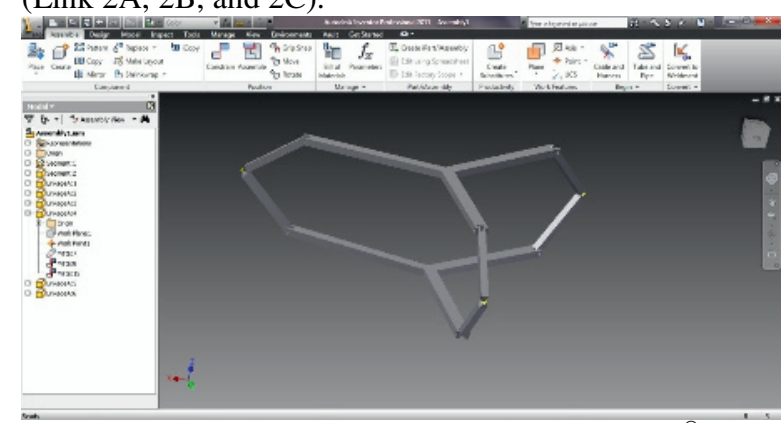

Fig. 3. 3D model of a segment created in Inventor ${ }^{\circledR}$.

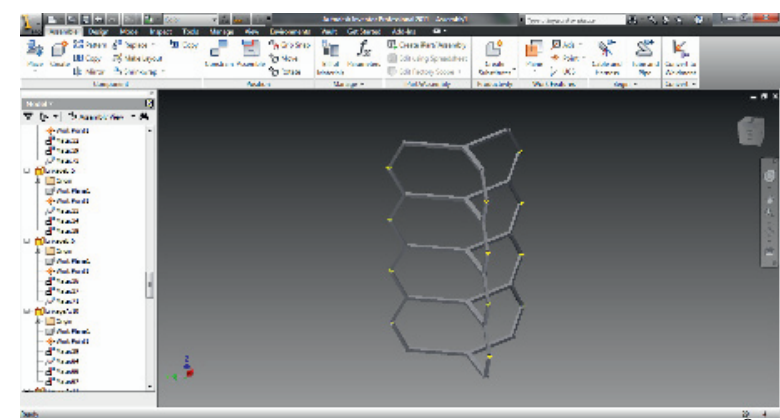

Fig. 4. 3D model of the serpentine robot created in Inventor ${ }^{\mathrm{B}}$.

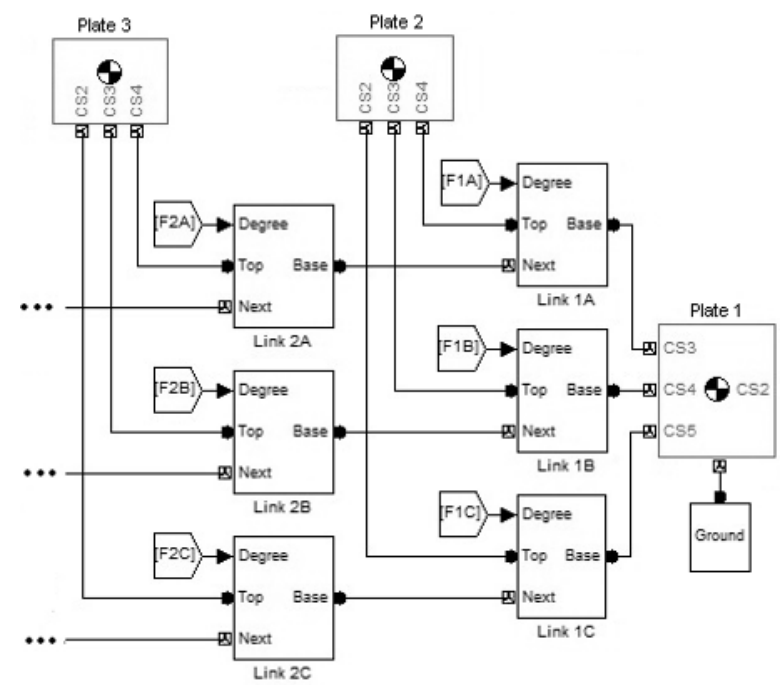

Fig. 5. Portion of the forward kinematic model of the serpentine robot in SimMechanics ${ }^{\circledR}$.

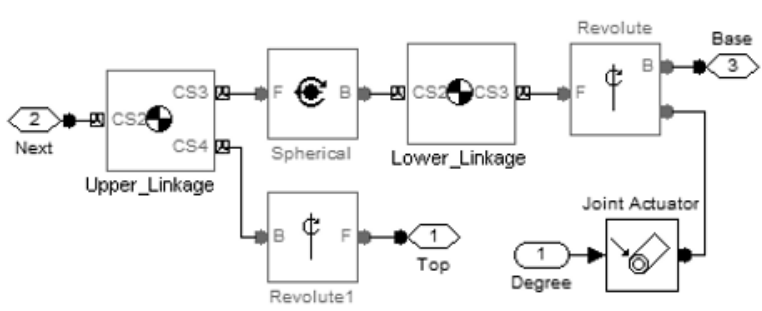

Fig. 6. Model of each folding linkage in each segment of the serpentine robot in SimMechanics ${ }^{\circledR}$. 
The detail model of each folding linkage is shown in Fig 6. Each folding linkage is consisted of the upper linkage and lower linkage which are connected by a spherical joint. The lower linkage is connected to the lower (base) plate through a revolute joint and the upper linkage is connected to the upper (top) plate. Upper end of the upper linkage is virtually at the same position of the lower end of the lower linkage of the next segment, therefor they are connected (through port Next).

At each segment the three angles $\alpha_{i}$ of the revolute joint between the lower lingkage and the lower plate can be given to the joint actuator. The position of all plates can be calculated given the set of angles $\alpha_{i}$ at each segment, hence enabling forward kinematic of the serpentine robot.

In order to calculate the set of angles $\alpha_{i}$ at each segment, we used the inverse kinematic equation solved in [5].

\section{Linear expansion rectilinear gait}

The three parallel actuators design gives the segment three degrees of freedom, which are rotation around the $\mathrm{x}$ and $y$ axes and linear translation along the $\mathrm{z}$ axis. The linear translation allows the segment to lengthen or shorten the distance between two plates. The other two degrees of freedom enable the serpentine robot to bend lateraly for turning even when the body of the robot is rotated around its length. This means that the serpentine robot can still move even when it is flipped around.

The linear expansion rectilinear gait adopted the stretching motion of the serpentine robot as described in [6]. However to simpify the gait design, we assume that the skin of the serpentine robot has a coefficient of friction of zero in the forward direction and infinite in the reverse direction. Therefore the robot does not have to raise its body in order to move its belly forward and does not slip when it pulls the belly backward. This is the approximation of the coefficient of friction between the scales on the belly of a snake and the ground.

In order for the serpentine robot to move forward it only has to expand/contract the length of the odd segments while contracting/expanding the length of the even segments respectively. Simply by repeating the expanding and contracting of the pair of segments accordingly will allow the serpentine robot to move straight forward continuously.

The movement sequence for straight forward movement is shown in Fig 7. In the first step the even segments expand by a certain distance while the odd segments contract by the same amount. Because the coefficient of friction is smaller in the forward direction some plates move forward while others stay. The normal distances of the segments are depicted by the gray dashed lines. In the second step the head and tail plates of the serpentine robots stay in position while the two middle plates move forward. The head and tail of the serpentine robot move forward again at the third step.

For forward movement while turning the segments have to rotate laterally while expanding or contracting.
Moreover, the expansion distance differs from the contraction distance.

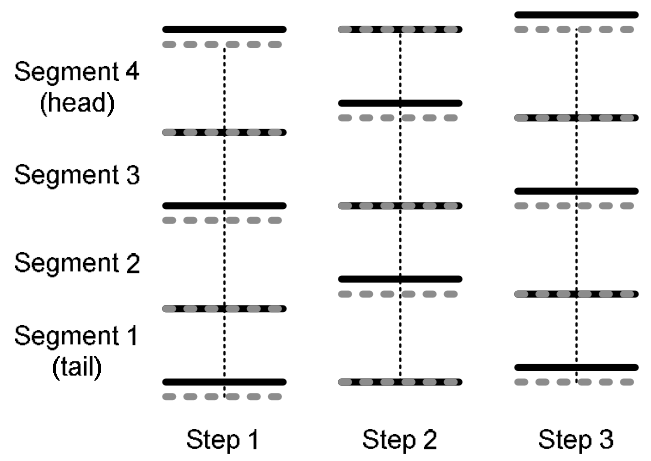

Fig. 7. Straight forward gait of the serpentine robot.

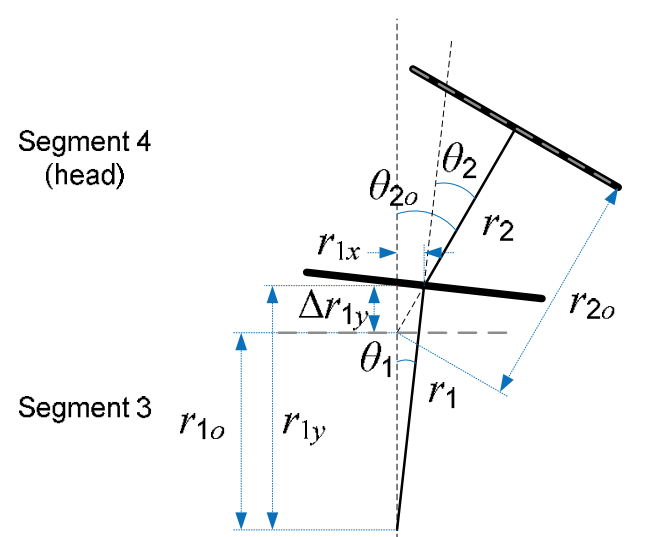

Fig. 8. The geometric of expanding and contracting of the segments for serpentine robot moving forward while turning.

The geometric for the expansion and contraction while turning is shown in Fig 8 where only two segments are shown, while the fourth segment is the head of the serpentine robot. The new angles of the third and fourth segment are $\theta_{1}$ and $\theta_{2}$ respectively after each step, while their previous angles are $\theta_{10}$ and $\theta_{20}$ respectively. Similarly the new lengths of the third and fourth segment are $r_{1}$ and $r_{2}$ respectively after each step, while their previous lengths are $r_{10}$ and $r_{20}$ respectively.

It can be shown that if the third segment change its length from $r_{1 o}$ to $r_{1}$ and the initial angle of the fourth segment with respect to the third segment is $\theta_{20}$, then the fourth segment has to change its length from $r_{20}$ to $r_{2}$ where

$$
r_{2}=r_{2 o}-\sqrt{r_{1 x}^{2}+\Delta r_{1 y}^{2}}
$$

The $3^{\text {rd }}$ segment also has to rotate from $\theta_{10}$ to $\theta_{1}$ where

$$
\begin{aligned}
& r_{1 y}=r_{1} \cos \left(\theta_{1}\right) \\
& \Delta r_{1 y}=r_{1} \cos \left(\theta_{1}\right)-r_{1 o}=r_{1} \cos \left(\theta_{1}\right)-A \\
& r_{1 x}=r_{1} \sin \left(\theta_{1}\right) \\
& \tan \left(\theta_{2 o}\right)=\frac{r_{1 x}}{\Delta r_{1 y}}=B \\
& B \cos \left(\theta_{1}\right)-\sin \left(\theta_{1}\right)=\frac{A B}{r_{1}}
\end{aligned}
$$


Solving the equation for $\theta_{1}$ yields

$$
\theta_{1}=\sin ^{-1} \frac{\left(r_{1 o} \tan \left(\theta_{2 o}\right)\right)}{ \pm r_{1} \sqrt{1+\tan \left(\theta_{2 o}\right)^{2}}}+\sin ^{-1}\left(\frac{-\tan \left(\theta_{2 o}\right)}{ \pm \sqrt{1+\tan \left(\theta_{2 o}\right)^{2}}}\right)
$$

The fourth segment has to rotate from $\theta_{2 \mathrm{o}}$ to $\theta_{2}$ where

$$
\theta_{2}=\theta_{2 \mathrm{o}}-\theta_{1}
$$

The simulation results of the forward while turning gait is shown in fig 9. The red segments expand while the black segments contract. It can be seen that the front plate stays in its place while the back plate moves forward, hence the front segments has to contract according to (1). To maintain the same direction, the angle of the fourth and third segments has to adjust their angle according to (2) and (3). The same calculation applies to the next two segments at the back. The first segment has to expand by the same amount as the third segment while the second segment has to contract according to (1). The two back segments also have to adjust their angles according to (2) and (3). When it is the turn for the fourth (head) segment to move forward, it can freely choose its heading angle. At the next step the other segments has to calculate their angles according to (2) and (3) to slither smoothly.

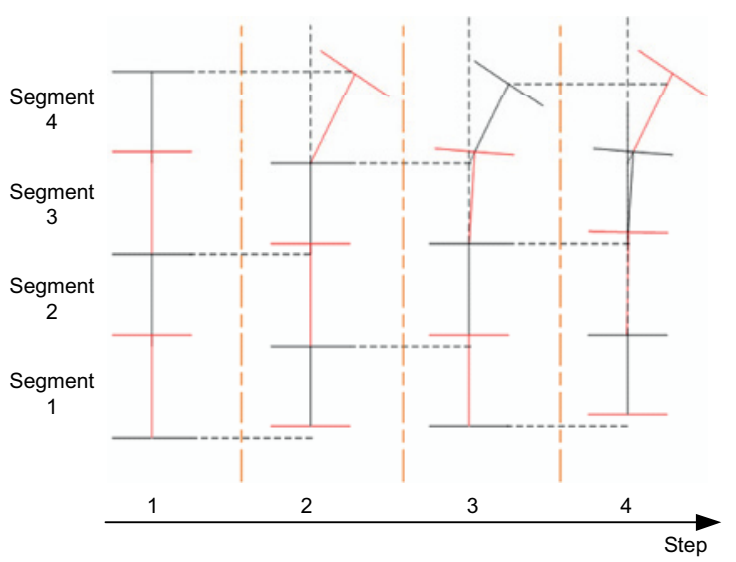

Fig. 9. The simulation result of forward-while-turning gait of the serpentine robot.

\section{Conclusion}

This article has shown the modeling a 3D structure of a serpentine robot with parallel actuators using the Autodesk Inventor ${ }^{\circledR}$ and then export the 3D model to SimMechanics ${ }^{\circledR}$ for kinematic modeling. It also compute the linear expansion gait for the serpentine robot for straight forward movement and forward movement while turning. The turning gait allows the head of the serpentine robot to turn freely while the back segments can follow smoothly for movement in confined spaces. This gait can be usefull for serpentine robot designed to aid urban search and rescue teams in order search into the voids in the rubble of disaster for victims.
Current research only assumes that the serpentine robot is moving in a planar surface. The same strategy can be used to expand the gait to move forward while turning laterally and vertically.

\section{References}

1 Murphy, R. R. Trial By Fire. IEEE Robotics and Automation Magazine, 11, 3, 50-61 (2004).

2 Hopkins, J. K., Spranklin, B., \& Gupta, S.. A survey of Snake-inspired Robot Designs, 4, 2 (2009).

3 Kinagasa, T., Otani, I., Haji, T., Yoshida, K., Osuka, K., \& Amano, H. Flexible Monothread Mobile Track (FMT) - A new Mobile Mechanism Using One Track and Vertebral Structure-. Robotics 2010: Current and Future Challenges, 241-259 (2010).

4 Borenstein, J., Hansen, M., \& Borrell, A. M. The OmniThread OT-4 Serpentine Robot - Design and Performance. Journal of Field Robotics, 601-621 (2007).

5 Behrens, R., Kuchler, C., Forster, T., \& Elkmann, N. Kinematik Analysis of a 3-DOF Joint for a Novel Hyper-Redundant Robot Arm. IEEE International Conference on Robotics and Automation, 3224-3229 (2011).

6 Hidetaka Ohno, Shigeo Hirose. Design of Slim Slime Robot and its Gait of Locomotion. IEEE/RSJ International Conference on Intelligent Robots and Systems, 707-715 (2001). 